\title{
COMPARING WHITE NOISE AND COLORED NOISE EFFECTS ON RLCG ELECTRICAL CIRCUITS
}

\author{
Edita KOLÁŘOVÁ*, Lubomír BRANČÍI*** \\ *Deptartment of Mathematics, Faculty of Electrical Engineering and Communication, Brno University of Technology, Brno, \\ Czech Republic, E-mail: kolara@ feec.vutbr.cz \\ **Department of Radio Electronics, Faculty of Electrical Engineering and Communication, Brno University of Technology, Brno, \\ Czech Republic, E-mail: brancik@feec.vutbr.cz
}

\begin{abstract}
We deal with RLCG electrical circuits with randomly varied potential source parameter. To model the situation we use the theory of stochastic differential equations, namely the Itô stochastic calculus. Our aim is to find the differences between the influences of the white noise and the colored noise on RLCG electrical circuit. In our examples we compute and illustrate the deterministic solutions and the corresponding confidence intervals for the stochastic solutions. Numerical simulations in the examples are carried out using Matlab.
\end{abstract}

Keywords: RLCG electrical network, stochastic differential equations, white noise, colored noise, stochastic numerical simulations

\section{INTRODUCTION}

We can describe systems, where the influence of the randomness is not negligible, by stochastic differential equations, where the randomness is represented by the white noise process. The effect of the white noise on RL circuits is examined in [1] and [2]. We have also examined RLC and RLCG circuits with coefficients influenced by the white noise. By application of the Itô calculus we have found analytic solutions as well as confidence intervals for the stochastic solutions, see [3]. The extension of this theory to higher order networks is presented in [4] and [5]. In all of this papers we worked only with white noise effected networks. However, in some situations the white noise doesn't sufficiently approximate the behavior of the noisy parameter of the circuit. In this situations we can use other processes with wide spectral bands (colored noise processes). First order electrical circuits with colored noise are the subject of [6]. In this paper we examine for the first time the effect of the colored noise on higher order electrical circuits. We adopted the Euler numerical scheme to find the stochastic trajectories of the colored noise effected circuit and to be able to compute confidence intervals for the solutions.

\section{EQUATIONS WITH RANDOMNESS}

Our method of involving randomness to physical systems is based on theory of stochastic differential equations. We start with an ordinary differential equation describing some physical system:

$\frac{\mathrm{d} y(t)}{\mathrm{d} t}=A(t, y(t)), y(0)=y_{0}$.

To involve randomness to the system, we add the term " $B(t, y(t)) \cdot$ noise" to the righthand side of the equation and multiple it by $\mathrm{d} t$. We get

$\mathrm{d} y(t)=A(t, y(t)) \mathrm{d} t+B(t, y(t)) \cdot$ noise $\cdot \mathrm{d} t$.

Now the solution of the system, $y(t)$, includes some randomness. We will call it stochastic process and denote it by capital letter, to distinguish it from the original deterministic function. Next we establish " $\mathrm{d} X(t)=$ noise $\cdot \mathrm{d} t$ ", where $X(t)$ is a stochastic process. We can mathematically describe the process $X(t)$ and involve it into the equation. We get

$\mathrm{d} Y(t)=A(t, Y(t)) \mathrm{d} t+B(t, Y(t)) \mathrm{d} X(t)$.

Considering the "noise" as the so called white noise process, we have $X(t)=W(t)$, where $W(t)$ is the Wiener process - a continuous process with independent increments, satisfying $W(0)=0$ and $W(t)-W(s)$ distributed $N(0, t-s)$, and we get the stochastic differential equation

$\mathrm{d} Y(t)=A(t, Y(t)) \mathrm{d} t+B(t, Y(t)) \mathrm{d} W(t), \quad Y(0)=Y_{0}$,

where $A:\langle 0, T\rangle \times \mathbb{R} \rightarrow \mathbb{R}$ is the drift coefficient and $B$ : $\langle 0, T\rangle \times \mathbb{R} \rightarrow \mathbb{R}$ is the diffusion coefficient, $Y_{0}$ is a random variable and the solution $Y(t)$ a stochastic process. We consider this equation in the integral form using the Itô stochastic calculus (see [7]). We can define similarly stochastic differential equations in multidimensional case (see again [7]).

In case of the colored noise, the process $X(t)$ satisfies the historically oldest example of a stochastic differential equation, the Langevin's equation (see [8]):

$$
\mathrm{d} X(t)=-\beta X(t) \mathrm{d} t+\sigma \mathrm{d} W(t), \quad X(0)=X_{0} .
$$

We can consider this as a model of the Brownian motion of a particle under the influence of friction but no other force field. Here $\beta>0$ is the coefficient of the friction, $\sigma$ is the diffusion coefficient and $X(t)$ is the velocity of the particle. Using the Itô formula we can find the analytic solution of the Langevin's equation $X(t)$ (see [9]), that is called the Ornstein-Uhlenbeck process:

$X(t)=e^{-\beta t} X_{0}+\sigma \int_{0}^{t} e^{-\beta(t-s)} \mathrm{d} W(s)$,

where the integral with respect to the Wiener process is the so called Itô integral (see [7]). We assume, that the moments $E\left[X_{0}\right]<\infty$ and $E\left[X_{0}^{2}\right]<\infty$. As the expectation of the Itô integral is zero, for the expectation of the OrnsteinUhlenbeck process we have:

$E[X(t)]=e^{-\beta t} \cdot E\left[X_{0}\right]$ 
Using the Itô isometry we can compute the second moment of $X(t)$ as well (see [9]):

$E\left[X^{2}(t)\right]=e^{-2 \beta t} \cdot E\left[X_{0}^{2}\right]+\frac{\sigma^{2}}{2 \beta}\left(1-e^{-2 \beta t}\right)$.

Thus the variance $V[X(t)]=E\left[X^{2}(t)\right]-E[X(t)]^{2}$ is

$V[X(t)]=e^{-2 \beta t} \cdot V\left[X_{0}\right]+\frac{\sigma^{2}}{2 \beta}\left(1-e^{-2 \beta t}\right)$.

We can see, that $E[X(t)] \rightarrow 0$ and $V[X(t)] \rightarrow \frac{\sigma^{2}}{2 \beta}$ as $t \rightarrow \infty$, and the distribution of $X(t)$ approaches $N\left(0, \frac{\sigma^{2}}{2 \beta}\right)$ for large $t$.

If we extend the equation (5) for the constant $\beta=0$, we get $X(t)=\sigma W(t)$. In that special case the OrnsteinUhlenbeck process is a multiple of the Brownian motion (or the Wiener process in mathematical notation). The expectation of the Wiener process is $E[W(t)]=0$ and the second moment is $E\left[W^{2}(t)\right]=t$ for all $t>0$.

To illustrate the diference, we picture some trajectories of the Wiener process, see Fig. 1 and some trajectories of the Ornstein-Uhlenbeck process, see Fig. 2 and Fig. 3.

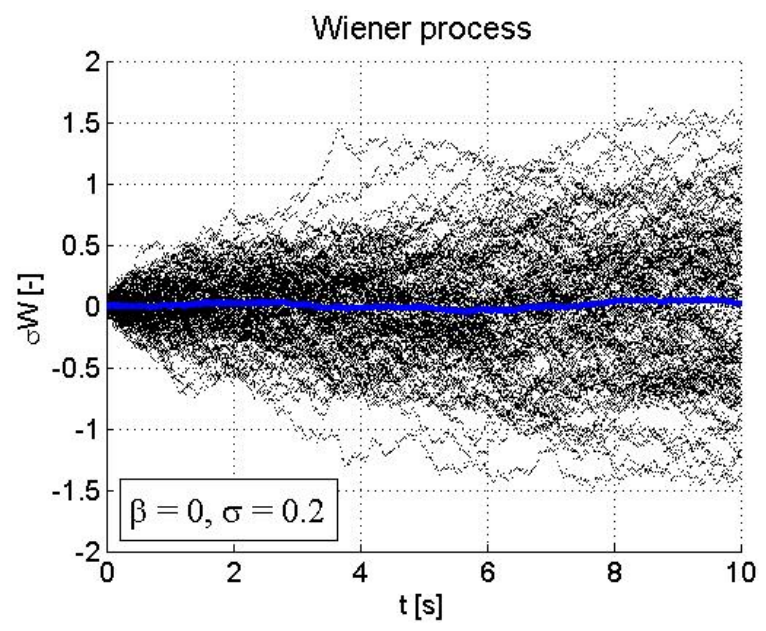

Fig. 1 Trajectories of the Wiener process

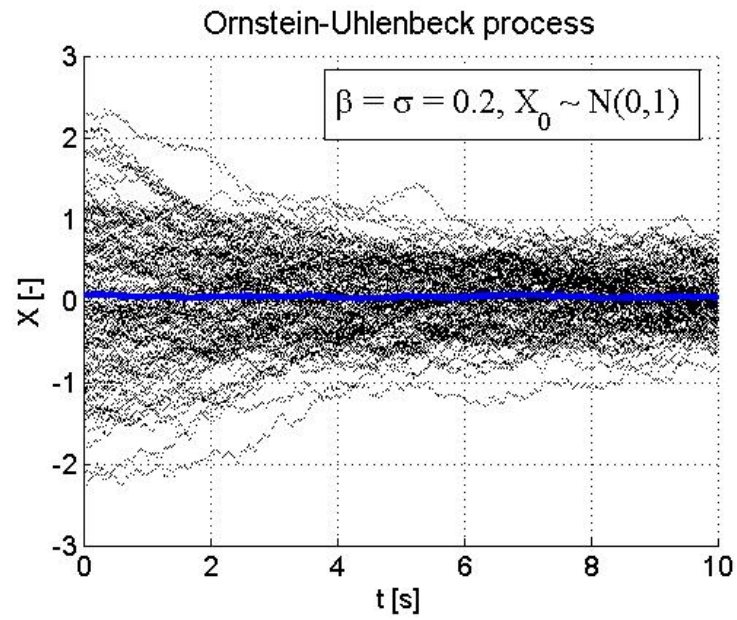

Fig. 2 Trajectories of the Ornstein - Uhlenbeck process with $N(0,1)$ distributed initial condition

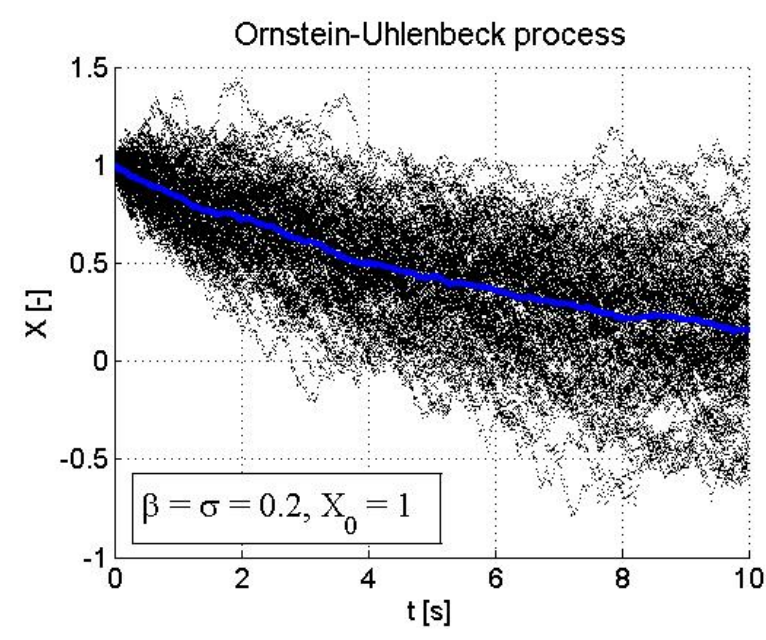

Fig. 3 Trajectories of the Ornstein - Uhlenbeck process with initial value $X_{0}=1$

\section{RLCG CIRCUIT MODEL}

\subsection{Deterministic second order RLCG circuit model}

We denote the parameters of the RLCG circuit as $L$ the inductance, $R$ the resistance, $G$ the conductance, $C$ the capacitance and $v(t)$ the potential source at time $t$. Then the capacitor voltage $v_{C}(t)$ and the inductor current $i_{L}(t)$ in the circuit satisfy the following system of ordinary differential equations

$\frac{\mathrm{d} v_{C}(t)}{\mathrm{d} t}=-\frac{G}{C} v_{C}(t)+\frac{1}{C} i_{L}(t)$,

$\frac{\mathrm{d} i_{L}(t)}{\mathrm{d} t}=-\frac{1}{L} v_{C}(t)-\frac{R}{L} i_{L}(t)+\frac{1}{L} v(t)$,

where initial conditions $v_{C}(0)=0, i_{L}(0)=0$ are considered. For our purpose it is more suitable to rewrite the equation into matrix form and multiply by $\mathrm{d} t$ :

$$
\mathrm{d} \mathbf{y}(t)=(\mathbf{A} \cdot \mathbf{y}(t)+\mathbf{a}(t)) \mathrm{d} t, \mathbf{y}(0)=\mathbf{c},
$$

where

$$
\begin{aligned}
& \mathbf{y}(t)=\left(\begin{array}{c}
y_{1}(t) \\
y_{2}(t)
\end{array}\right)=\left(\begin{array}{c}
v_{C}(t) \\
i_{L}(t)
\end{array}\right) \\
& \mathbf{A}=\left(\begin{array}{cc}
-\frac{G}{C} & \frac{1}{C} \\
-\frac{1}{L} & -\frac{R}{L}
\end{array}\right), \mathbf{a}(t)=\left(\begin{array}{c}
0 \\
\frac{v(t)}{L}
\end{array}\right), \mathbf{c}=\left(\begin{array}{c}
v_{C}(0) \\
i_{L}(0)
\end{array}\right) .
\end{aligned}
$$

The solution of $(12)$ is

$$
\mathbf{y}(t)=\mathrm{e}^{\mathbf{A} t} \mathbf{c}+\int_{0}^{t} \mathrm{e}^{\mathbf{A}(t-s)} \mathbf{a}(s) \mathrm{d} s .
$$




\subsection{Stochastic RLCG circuit model}

To create a stochastic model, we consider the potential source in RLCG circuit influenced by random effects. From now on the output of the circuit would be a stochastic process, therefore we introduce capital $Y$ as a new notation for the solution (13).

1.) First we replace $v(t)$ in deterministic model $(11)$ by $v(t)+$ "white noise". After the computations described in chapter2 we get the stochastic differential equation

$\mathrm{d} \mathbf{Y}(t)=(\mathbf{A} \cdot \mathbf{Y}(t)+\mathbf{a}(t)) \mathrm{d} t+\mathbf{b} \mathrm{d} W(t), \quad \mathbf{Y}(0)=\mathbf{c}$,

where $\mathbf{b}=\left(\begin{array}{c}0 \\ \frac{\sigma}{L}\end{array}\right)$ and $\sigma$ is the intensity of the noise.

In [3] we solved this equation analytically and computed the expectation and the second moment of the stochastic solution. If the initial values $v_{C}(0)$ and $i_{L}(0)$ are constant, the expectation of the stochastic solution $E[\mathbf{Y}(t)]$ is equal to the deterministic solution (12).

2.) This time we replace $v(t)$ in 11 by $v(t)+$ "colored noise". After some computations, see chapter 2 , we get

$$
\mathrm{d} \mathbf{Y}(t)=(\mathbf{A} \cdot \mathbf{Y}(t)+\mathbf{a}(t)) \mathrm{d} t+\mathbf{b} \mathrm{d} X(t), \quad \mathbf{b}=\left(\begin{array}{c}
0 \\
\frac{1}{L}
\end{array}\right),
$$

$\mathbf{Y}(0)=\mathbf{c}, \quad \mathrm{d} X(t)=-\beta X(t) \mathrm{d} t+\sigma \mathrm{d} W(t), \quad X(0)=X_{0}$.

$\beta, \sigma$ are constants, determining the character and the intensity of the noise and $W(t)$ is the Wiener process.

\section{NUMERICAL SOLUTIONS}

To solve the equations $(16)-(17)$ we use numerical techniques. We will use the stochastic Euler numerical scheme (see [10]), which is derived from the Euler method for ordinary differential equations. Let $N>0, N \in \mathbb{N}$. We define an equidistant discretisation of the interval $\langle 0, T\rangle$ as:

$t_{n}=n h$ for $n=0, \ldots, N$, where $h=\frac{T}{N}=t_{n+1}-t_{n}$,

and generate $\Delta W_{n}=W\left(t_{n+1}\right)-W\left(t_{n}\right)=\int_{t_{n}}^{t_{n+1}} \mathrm{~d} W(s)$, as independent Gauss random variables with $E\left[\Delta W_{n}\right]=0$ and $E\left[\left(\Delta W_{n}\right)^{2}\right]=h$. Now we can use the Euler scheme to compute the increments of the colored noise process as

$\Delta X_{n}=X_{n+1}-X_{n}=-\beta X_{n} h+\sigma \Delta W_{n}$.

We are now ready to apply an implementation of the Euler method to the equation 16 with initial value $\mathbf{Y}(0)=\mathbf{c}=$ $\mathbf{Y}_{0}$.

$\mathbf{Y}_{n+1}=\mathbf{Y}_{n}+\left(\mathbf{A} \mathbf{Y}_{n}+\mathbf{a}\left(t_{n}\right)\right) h+\mathbf{b} \Delta X_{n}$

Here the scheme is written in vector form.

\section{EXAMPLE}

We work on the model of the RLCG circuit, see Fig. 4 with all parameters unit.

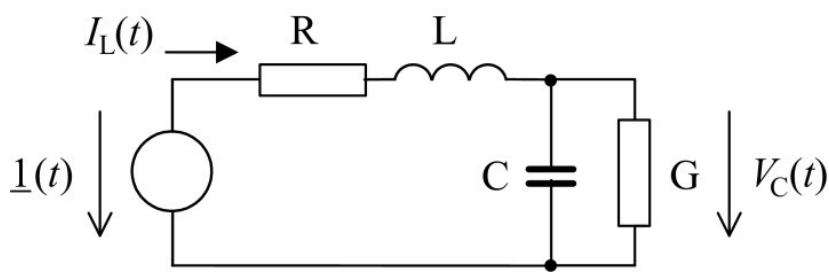

Fig. 4 RLCG circuit

Let us consider the voltage source influenced by randomness:

$\underline{1}^{*}(t)=\underline{1}(t)+"$ noise",

with remaining circuit parameters deterministic. We consider zero initial conditions in this example.

Our goal is to illustrate the effects of different types of "noise" in the potential source on the solution of the circuit. The mathematical representation of the noise is the solution of (17) with different coefficients $\sigma$ and $\beta \geq 0$ and with different initial conditions of the colored noise process.

To illustrate the effect of the noise, we generate a large amount of trajectories and compute statistically confidence intervals for the mean and for the stochastic solutions using the Student's t-distribution.

a) First we consider the "noise" in the voltage source equal to the white noise, which is represented by the Wiener process. In Fig. 5 and 6 we pictured the $99 \%$ confidence interval for the nhelan and for the stochastic solutions (the dashed lines on the pictures).

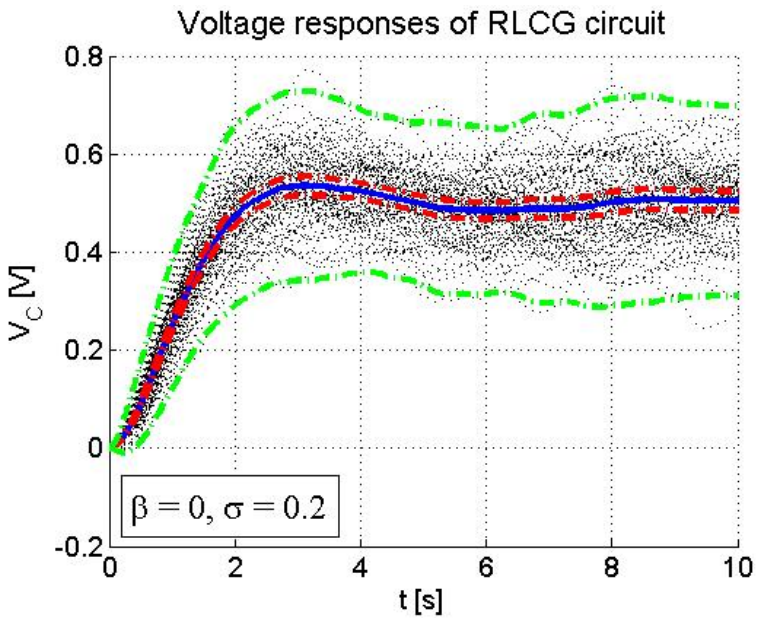

Fig. 5 Stochastic voltage responses in RLCG circuit with white noise influenced source 


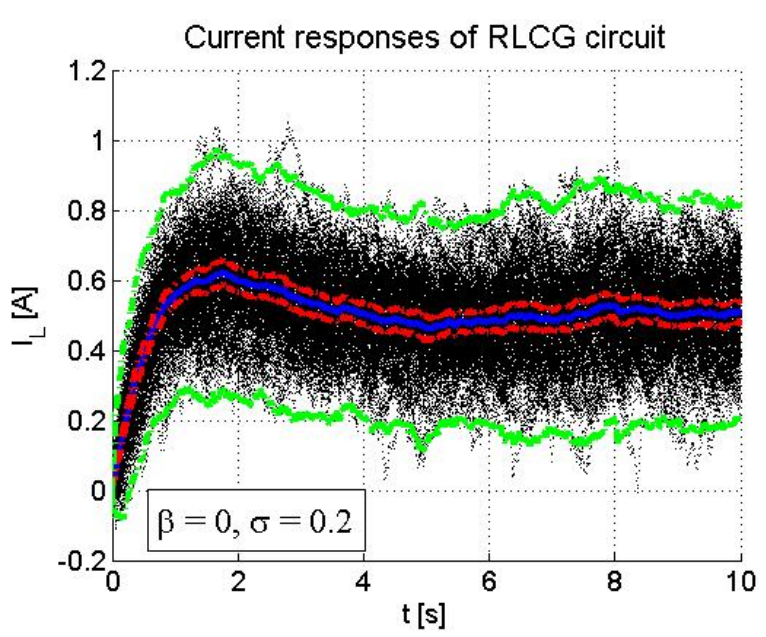

Fig. 6 Stochastic current responses in RLCG circuit with white noise influenced source

b) This time we consider the "noise" in the source as colored noise, represented by Ornstein-Uhlenbeck process with zero initial condition. In Fig. 7 and 8 we picture the trajectories of the stochastic solutions and the corresponding confidence intervals.

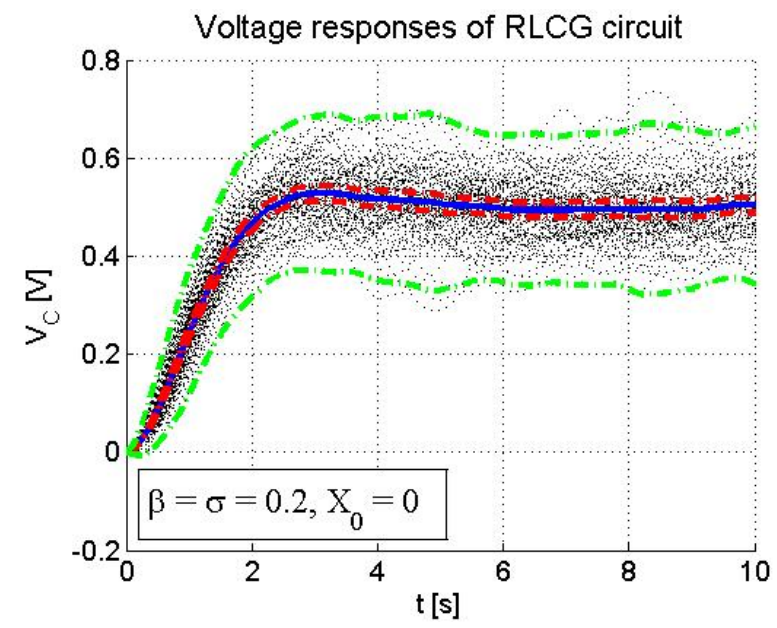

Fig. 7 Stochastic voltage responses with colored noise influenced source

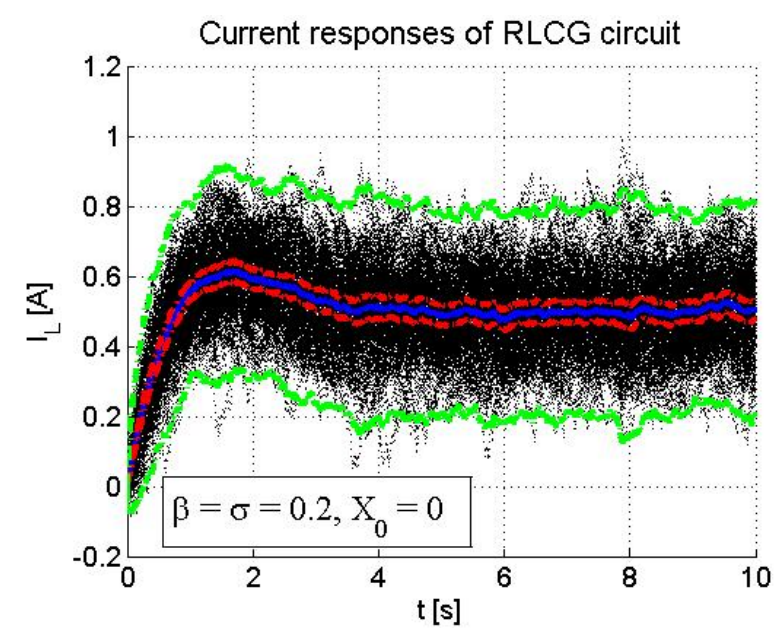

Fig. 8 Stochastic current responses with colored noise influenced source c) Now the "noise" in the voltage source is again colored noise, represented by the Ornstein-Uhlenbeck process with normally distributed initial condition. See the trajectories of the stochastic solution and the corresponding confidence intervals in Fig. 9 and 10.

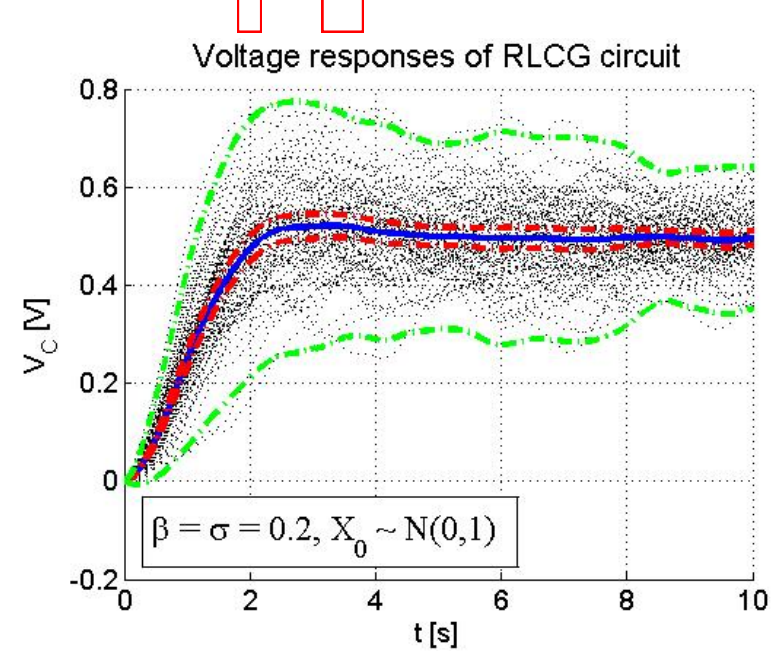

Fig. 9 Stochastic voltage responses with colored noise influenced source, $X_{0} \sim \mathrm{N}(0,1)$ distributed random variable

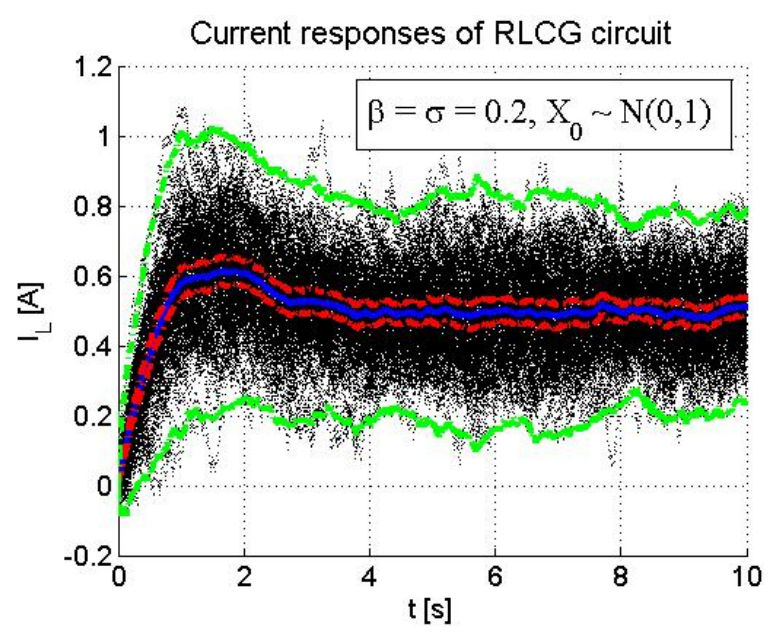

Fig. 10 Stochastic current responses with colored noise influenced source, $X_{0} \sim \mathrm{N}(0,1)$ distributed random variable

d) In the Fig. 11 and 12 we pictured the trajectories of the stochastic solutions and the corresponding confidence intervals when the voltage source in the RLCG circuit is influenced by colored noise, represented by OrnsteinUhlenbeck process with unit initial condition.

Here the expectation of the noise representation is $E[X(t)]=e^{-0,2 t}$, which is not zero as in the examples, we had before. That's why in this case the expectation of the stochastic solution does not equal to the deterministic solution. For increasing $t$ the expectation $E[X(t)]$ goes to zero, and so the stochastic voltage and current responses go to the deterministic solutions of the circuit with $v(t)=\underline{1}(t)$ (no noise in potential source). 


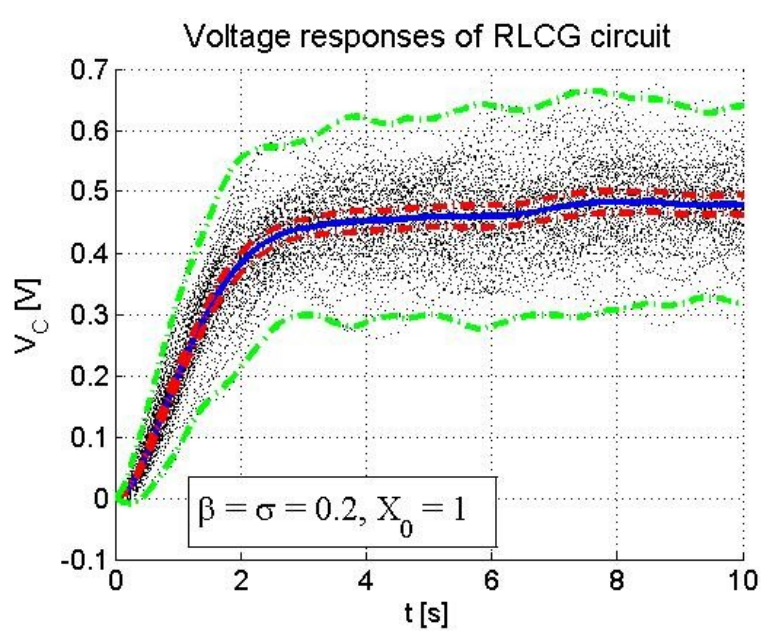

Fig. 11 Stochastic voltage responses with colored noise influenced source, $X_{0}=1$

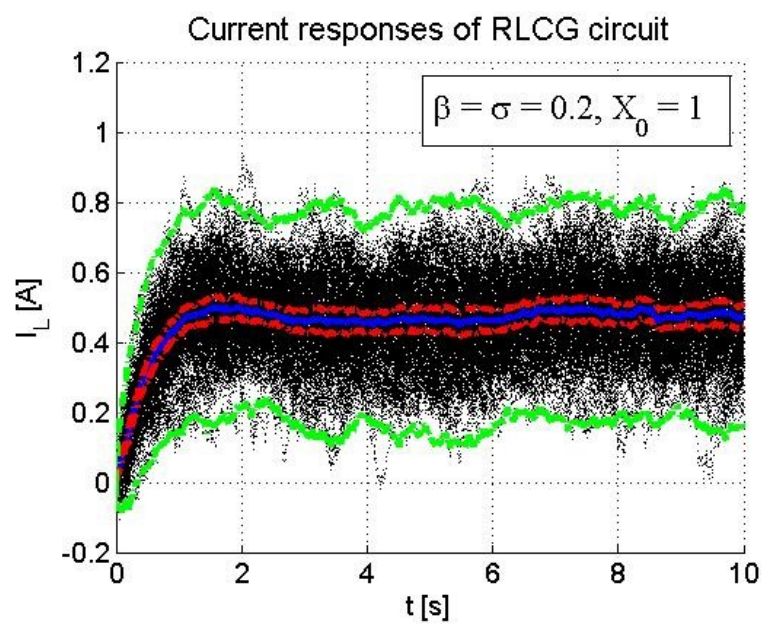

Fig. 12 Stochastic current responses with colored noise influenced source, $X_{0}=1$

\section{CONCLUSION}

In the theory of stochastic differential equations the randomness is considered as the white noise. Here we work with systems influenced by a colored noise as well. This type of modelling can be very useful for real systems, where the colored noise occurs. As an example we can mention the pink noise in semiconductors, where a non-linear SDE would be solved in general, or a noise small-signal linearized model of a device considered. We illustrated the effect of the noise in source on the solution of the RLCG circuit in our example. While in the white noise effected circuit the expectation of the stochastic solution is equal to the deterministic one, in the colored noise effected circuit it can be different, but for increasing $t$ the differences between the stochastic solutions of the colored noise effected circuit and the white noise effected circuit diminish.

\section{ACKNOWLEDGEMENTS}

This work was supported by Czech Science Foundation under grant 15-18288S. For research, infrastructure of the SIX Center was used.

Received June 30, 2016, accepted July 28, 2016

\section{REFERENCES}

[1] KOLÁŘOVÁ, E.: Statistical estimates of stochastic solutions of RL electrical circuits, in Proc. of the IEEE International Conference on Industrial Technology ICIT06, Mumbai, pp. 2546-2550, 2006.

[2] KOLÁŘOVÁ, E.: An application of stochastic integral equations to electrical networks, in Acta Electrotechnica et Informatica, vol. 8, no. 3, pp. 14-17, 2008.

[3] KOLÁŘOVÁ, E.: Applications of second order stochastic integral equations to electrical networks, in Tatra Mountains Mathematical Publications, vol. 63, pp. 163-173, 2015.

[4] BRANČÍK, L. - KOLÁŘOVÁ, E.: Simulation of higher-order electrical circuits with stochastic parameters via SDEs, Advances in Electrical and Computer Engineering, vol. 13, no. 1, pp. 17-22, 2013.

[5] BRANČÍK, L. - KOLÁŘOVÁ, E.: Application of Stochastic Differential-Algebraic Equations in Hybrid MTL Systems Analysis" Elektronika ir Elektrotechnika, vol. 20, no. 5, pp. 41-45, 2014.

[6] RAHMAN, F. - PARISA, N.: A stochastic perspective of RL electrical circuit using different noise terms, COMPEL: The International Journal for Computation and Mathematics in Electrical and Electronic Engineering, vol. 30, no. 2, pp. 812-822, 2011.

[7] ØKSENDAL, B.: Stochastic Differential Equations, An Introduction with Applications, Springer-Verlag, 2000.

[8] ARNOLD, L.: Stochastic Differential Equations: Theory and Applications, New York: John Wiley \& Sons, 1974.

[9] KOLÁŘOVÁ, E.: The Langevin's equation as a stochastic model of an electrical circuit, in Poc. of the XXV International Colloquium,Brno, 2007.

[10] KLOEDEN, P. - PLATEN, E. - SCHURZ, H.: Numerical Solution of SDE Through Computer Experiments, New York: Springer-Verlag, 1997.

\section{BIOGRAPHIES}

Edita Kolářová was born on 22. 6. 1965. In 1988 she graduated in mathematics at the Faculty of Mathematics and Physics, Charles University, Prague. She defended her PhD in the field mathematics in engineering in 2006. She is working as an associated professor at the Department of Mathematics at Brno University of Technology. Her scientific research is focusing on applications of stochastic differential equations to electrical network.

Lubomír Brančík was born on 29. 3. 1961. He studied microelectronics and graduated in 1985, then received his CSc (PhD equivalent) degree in measurements in 1993, both in Brno University of Technology. He is currently a professor for theoretical electrical engineering at the Department of Radio Electronics, Brno University of Technology. His research interest is in numerical methods for the analysis of electronic circuits, computer-aided simulations, and signal integrity issues in mixed electronic circuits. 